\title{
Does diet have a role in the aetiology of rheumatoid arthritis?
}

\author{
Dorothy J. Pattison ${ }^{1 *}$, Deborah P. M. Symmons ${ }^{1}$ and Adam Young ${ }^{2}$ \\ ${ }^{1}$ Arthritis Research Campaign Epidemiology Unit, Stopford Building, University of Manchester, \\ Oxford Road, Manchester M13 9PT, UK \\ ${ }^{2}$ Department of Rheumatology, City Hospital, St Albans AL3 5PN, UK
}

\begin{abstract}
Although dietary factors have been extensively studied in many chronic diseases, the role of diet in the epidemiology of rheumatoid arthritis (RA) has received little attention. Fruit and vegetables and dietary antioxidants are thought to play a protective role in the pathogenesis of CVD and some cancers, but few studies have investigated these dietary components in the aetiology of RA. Fish oil supplementation has consistently been shown to have a beneficial effect on the symptoms of established RA, but it is not known whether the PUFA present in fish oils can reduce the risk of developing the disease. There is evidence that RA is less severe in the southern Mediterranean countries, such as Italy and Greece, where oil-rich fish, fruit, vegetables and olive oil are consumed in greater amounts than in many other countries. Overall, the evidence for a role of diet in the aetiology of RA is limited to a small number of observational studies of very different designs. Recently, it was demonstrated that lower intakes of fruit and vegetables and dietary vitamin $\mathrm{C}$ are associated with an increased risk of developing inflammatory polyarthritis in a free-living population in Norfolk, UK. These findings provide further evidence for a role of diet in the development of inflammatory arthritis, although the mechanisms involved are uncertain.
\end{abstract}

Rheumatoid arthritis: Diet: Risk factor

Rheumatoid arthritis (RA) is the most common form of inflammatory polyarthritis (IP), with a worldwide prevalence in adults of approximately $1 \%$ (Abdel-Nasser et al. 1997). RA affects women more commonly than men by a ratio of $3: 1$, and although the peak age of onset in women is in the sixth decade, it can occur at any age. Its course varies considerably from a mild variant to a severe chronic deforming condition. Severe disability or marked functional loss can be a problem even in the early stages of the disease, affecting $\leq 10 \%$ of patients after 5 years of followup (Young et al. 2000). RA is associated with an increase in mortality from both complications of the disease and its treatment, and also from CVD, with a reported reduced life expectancy of between 3 and 10 years. Overall, RA results in enormous personal, social and economic costs to patients and the community.

\section{Aetiology}

The cause of RA remains uncertain, but genetic factors are known to be involved and the locus has been identified.
Which genetic factors play a part in susceptibility to, rather than the severity of, RA remains controversial. It has been suggested that genetic susceptibility explains $\leq 40 \%$ of the risk of developing RA (Silman et al. 1993). Environmental and non-genetic constitutional factors must therefore account for the main risks. Several putative antigens have been proposed (Table 1), the most likely being an infectious agent, but the evidence is inconclusive. It is likely that there is more than one potential environmental trigger of RA and that these factors may interact, resulting in additive effects (e.g. smoking plus infection).

\section{Characteristics of rheumatoid arthritis}

RA is characterised in the early stages by persistent inflammation of the synovial lining of the joints (Fig. 1), which can lead to the destruction of bone and cartilage and result in joint deformity. Typically, joints are affected symmetrically and small joints are involved earlier than large, i.e. axial, joints. The condition is often associated 
Table 1. Possible causes of rheumatoid arthiritis (Symmons \& Harrison, 2000)

\section{Risk factor}

Genetic, human leucocyte-associated antigen DRB4

Viral infection, e.g. Epstein-Barr virus, Proteus, rubella, parvovirus Immunisation, e.g. rubella, tetanus, influenza

Hormonal and reproductive factors, e.g. prolactin production during lactation

Smoking

Previous blood transfusion

Obesity

Stress or physical trauma

with systemic disturbance, most commonly fatigue, stiffness, anaemia and weight loss, and extra-articular features such as rheumatoid nodules. Two characteristics of the condition important in diagnosis are the presence of rheumatoid factor (RF) measured in blood, and typical RA erosions seen on radiological examination of hands and feet. RF is used both in diagnosis and prognosis, but lacks sensitivity because it is found in only 70-80\% of RA cases. It is also seen in approximately $5 \%$ of normal populations. It probably does not itself have a pathogenic role, but it is a marker of the individual's ability to produce self-associating complexes of Ig. IP describes a heterogeneous condition, with RF-positive, erosive and chronic disease at one end of the spectrum, when the diagnosis of RA is easily made, and RF-negative, non-erosive and often milder or self-limiting disease at the other end.

\section{Mechanism of inflammation}

Inflammation is the body's reaction to invasion by an infectious agent, antigen or physical damage. Antigen exposure triggers the immune response, resulting in a cascade of cellular activity and an inflammatory response in the end organ (Fig. 2). In RA the immune response is not switched off, the inflammatory response continues in articular tissue, as though in response to a persistent stimulus, leading in time to irreversible damage to tendons and joints. Although a greater understanding of efferent mechanisms of inflammation and tissue destruction in RA has evolved over the past 10 years (Arend, 2001), there is still little understanding as to why inflammation persists in RA.

\section{Free oxygen radicals and antioxidants}

Following initiation of the immune response, activated macrophages, monocytes and granulocytes generate free oxygen radicals (Halliwell et al. 1988). The rapidly proliferating cells of the immune system are uniquely prone to oxidative damage by free radicals and pro-inflammatory PG (PGE 2 ) and cytokines, in particular TNF- $\alpha$ and IL-1 (Kremer, 1993). Products of free radical oxidation have been identified in synovial fluid (Lunec et al. 1981; Merry et al. 1989), thus lending further support to the theory that RA itself may be, in part, mediated by free radical activity.

The exogenous antioxidant micronutrients vitamins $\mathrm{C}$ and $\mathrm{E}, \beta$-carotene and $\mathrm{Se}$ and other carotenoids such as lycopene, lutein and $\beta$-cryptoxanthin are efficient scavengers of free radicals. Increased lipid peroxidation and depletion of ascorbate in serum and synovial fluid provide evidence consistent with oxidative stress in rheumatoid disease, and it appears that the depletion of ascorbate is a result of oxidation during its antioxidant activity (Halliwell et al. 1988). Thus, antioxidant micronutrients may have an important role in preventing tissue damage caused by free oxygen radicals (Halliwell \& Gutteridge, 1999).

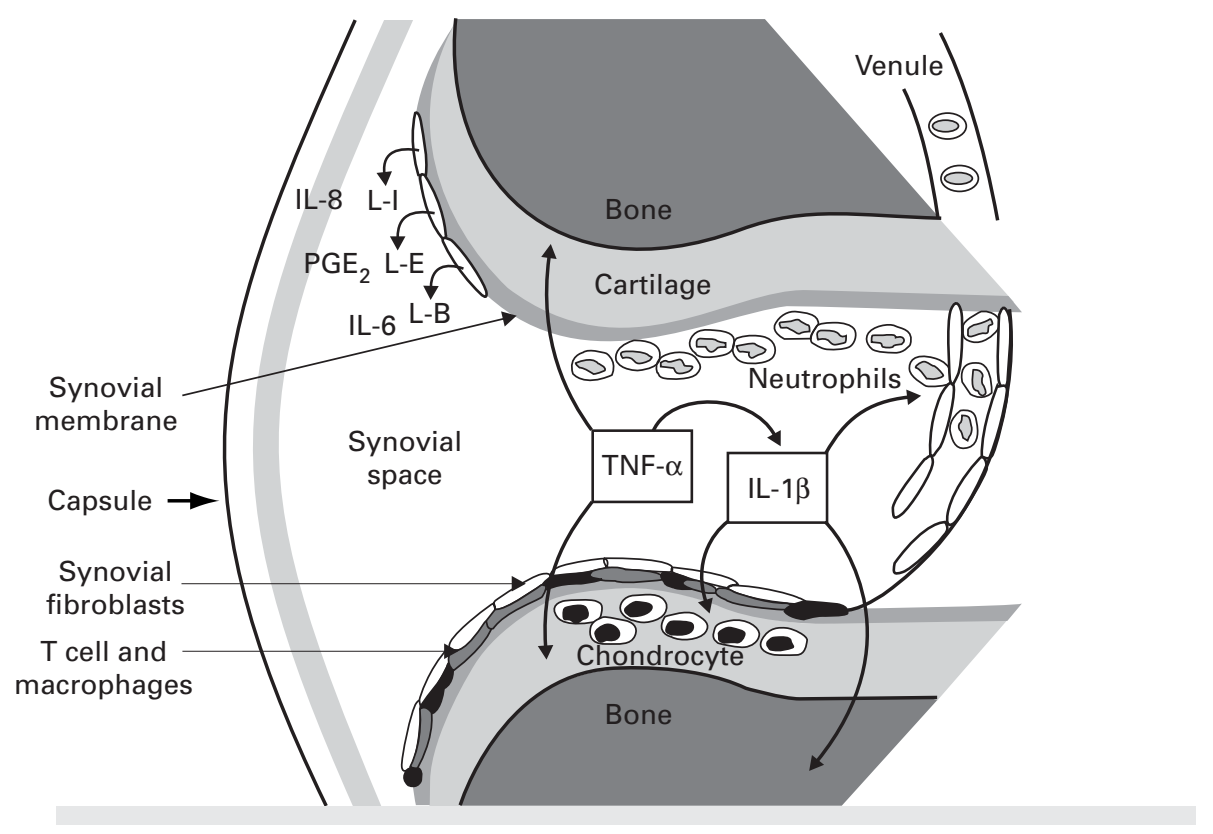

Fig. 1. Diagram to show the inflammatory process in the knee joint. 


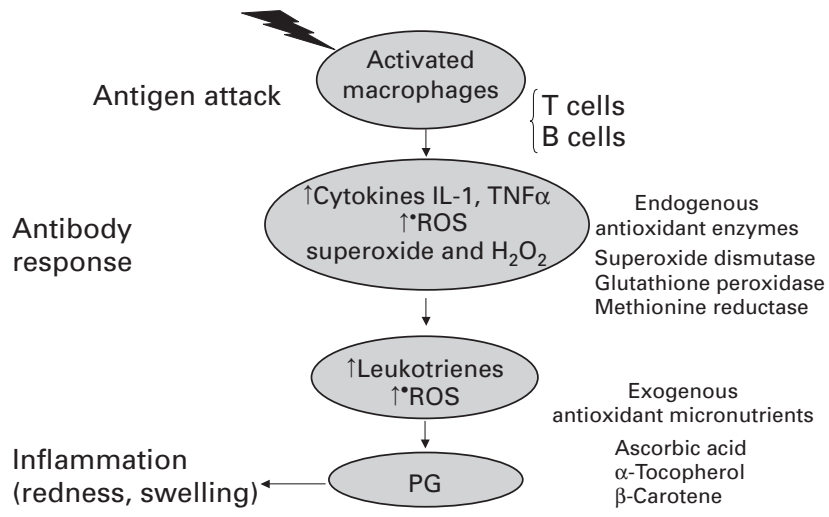

Fig. 2. Diagram to show the molecular pathway of the inflammatory process. ROS, reactive oxygen species.

\section{Is there any evidence that antioxidants are involved in the pathogenesis of rheumatoid arthritis?}

\section{Serum antioxidants}

Two 'nested' case-controlled studies from Finland provide some important information. Serum taken from participants in a national health survey between 1968 and 1972 was analysed for $\alpha$-tocopherol, $\beta$-carotene and Se levels. After 20 years of follow-up, fourteen incident cases of RA were identified and serum levels were compared with two controls matched for age and gender (Heliövaara et al. 1994). Lower serum levels of $\alpha$-tocopherol, $\beta$-carotene and Se (lowest tertile compared with highest tertile) were associated with a modest increase in the risk of developing RA. An 'antioxidant index' (defined as 'the product of the molar concentrations of $\alpha$-tocopherol, $\beta$-carotene and Se') had the strongest association, but this association was not maintained after adjusting for serum cholesterol level. Surveillance of individuals in a later health survey confirmed the inverse association between serum $\mathrm{Se}$ and $\alpha$-tocopherol levels and the risk of developing RA in a further 122 incident cases compared with age- and gendermatched controls (Knekt et al. 2000). However, serum Se levels were lower only in subjects with seronegative RA and the association between $\alpha$-tocopherol and RA onset was no longer significant after 10 years of follow-up. $\beta$-Carotene levels were not reported. US researchers also used a nested case-control design to investigate serum antioxidant concentrations using samples given by blood donors during 1974 (Comstock et al. 1997). Donors who went on to develop RA ( $n$ 21) had lower serum levels of $\alpha$-tocopherol, $\beta$-carotene and retinol compared with age-, gender- and race-matched controls (four controls per case). The RA cases had lower mean values than the controls for all three antioxidants, but only the difference for $\beta$-carotene reached significance $(-6 \cdot 9$ (95\% CI $-13 \cdot 1$, -0.6)). In all three studies serum samples had been stored for long periods of time, which may have affected the stability of the micronutrients, and given that serum levels of antioxidants may not reflect dietary intake accurately other than for $\beta$-carotene (Bates et al. 1997), these results can only suggest that antioxidants have a protective role but they are certainly not conclusive.
Low plasma levels of antioxidants have been demonstrated in prevalent cases of adult (Halliwell et al. 1988; Kiziltunç et al. 1998) and juvenile (Helgeland et al. 2000) RA, compared with healthy individuals. However, it is unclear whether this state exists pre-diagnosis, either as a result of low dietary intake or early antioxidant activity, or if it occurs as a consequence of established inflammatory disease.

\section{Dietary intake of antioxidants}

There has been little research into the dietary aetiology of RA and very little relating specifically to fruit and vegetable or antioxidant intake. Using data from the 1993 Italian Multipurpose Household Survey, La Vecchia et al. (1998) investigated the association between vegetable intake and the prevalence of twelve chronic diseases, including 'arthritis'. Participants estimated their weekly number of servings of vegetables (excluding potatoes and legumes) and the data were analysed according to less than seven, seven, more than seven servings per week. The authors did not define arthritis, but given that the prevalence was estimated at $232 \cdot 5 / 1000$ it is likely that this data includes any musculo-skeletal condition reported by participants. There was a significant inverse association between the highest category of vegetable intake and the risk of arthritis (odds ratio (OR) 0.84 (95\% CI 0.79, $0 \cdot 90)$ ).

Higher intakes of cooked, but not raw, vegetables were associated with a reduced risk of RA in a Greek hospitalbased case-control study (Linos et al. 1999). Consumption of cooked vegetables in the highest quartile of intake (two to three servings per d) compared with the lowest quartile of intake (less than one serving per $d$ ) reduced the risk of RA by about $60 \%$. More recently, Cerhan et al. (2003) found that lower intakes of cruciferous vegetables and $\beta$-cryptoxanthin may increase the risk of developing RA in older women participating in the Iowa Women's Health Study. Fruit intake was also weakly associated with risk of RA, suggesting that higher fruit intake confers a protective effect against RA onset. This study is the only one to specifically evaluate the dietary intake of fruit and antioxidants and the development of RA. However, a population-based case-control study principally designed to investigate fish intake and the risk of RA in women also analysed their data for various micronutrients. No association between vitamins $\mathrm{A}$ or $\mathrm{E}$ and the risk of RA were found, although a weak inverse relationship was observed for vitamin C (Shapiro et al. 1996). A higher consumption of fruit and vegetables was weakly, but not significantly, associated with a lower risk of developing RA.

\section{Other foods and nutrients associated with the development of rheumatoid arthritis}

In the pre-war years it was recognised that several chronic diseases developed as a result of nutritional deficiency. Scurvy was found to be a consequence of vitamin $\mathrm{C}$ deficiency, beriberi was a deficiency of thiamine and pellagra was a deficiency of niacin. RA was generally thought to be of infectious aetiology and it was widely believed that any evidence found of a food-deficiency state 
in RA was secondary to the disease. However, two studies (Hall \& Myers, 1935; Bayles et al. 1943) attempted to assess dietary intake before the onset of arthritis but could not identify a direct relationship. Hall \& Myers (1935) evaluated the dietary histories of seventy-five cases of 'chronic arthritis,' and forty patients with atrophic (rheumatoid) arthritis in a hospital-based 'case-control' study. Thirty medical patients (with a similar age, gender and social-class distribution to the cases) with a variety of respiratory and other conditions acted as controls. Dietary assessment was carried out by interview. Both groups were considered to have equally poor dietary intakes, although low energy and vitamin intakes were found in a greater number of patients in the atrophic arthritis group compared with the other arthritis groups. Bayles et al. (1943) arrived at a similar conclusion from their survey of patients' diets before the onset of RA. They used a variety of sources of nutritional data as comparators, including reports by other authors, data on the average US diet in North Atlantic cities and the Nutrition Research Council recommendations for dietary intake. They concluded that patients' diets were not 'grossly different' from those of the comparators. Such early studies were limited by poor study design and the lack of availability of nutritional data at that time, and it is not possible to draw definite conclusions.

\section{Dietary fat}

There is consistent evidence that $n-3$ PUFA have a beneficial role in established RA (Rennie et al. 2003). Intervention studies have investigated the effect of fish oil supplementation, rather than dietary sources, on disease outcome rather than initiation (Fortin et al. 1995). Cytokines and PG (eicosanoids), which play a major role in mediating the immune response and inflammation, are derived from $n-3$ and $n-6$ PUFA found in cell membranes (Fig. 3). Those derived from $n-6$ PUFA are more pro-inflammatory than those derived from $n-3$ PUFA (Darlington \& Stone, 2001). A diet rich in $n-3$ PUFA can increase the proportion of $n-3$ PUFA contained in cell membranes and increase the production of the less-inflammatory eicosanoids, potentially helping to reduce pain and symptoms. However, there is much less evidence that long-chain fatty acids are involved in the onset of RA.
Three studies have specifically looked at the intake of plant and marine oils and the risk of developing RA. Two hospital-based case-control studies carried out by the same group in Greece (Linos et al. 1991, 1999) have demonstrated a strong negative association between greater olive oil consumption and the risk of RA. The first study (Linos et al. 1991) was performed with no a priori hypothesis and compared the dietary intake of 168 prevalent RA cases and 137 controls using a 100-item food-frequency questionnaire. As a large number of variables were investigated it is possible that the associations could be chance findings. Although olive oil consumed more than once per $d$, compared with six times per month, reduced the risk of developing RA (relative risk 0.26 (95\% CI 0.07, 0.98)), only four cases and eight controls consumed olive oil at the highest level of intake. A weak protective effect was also observed when fish consumption was evaluated as an independent risk factor. An interesting finding was the protective effect of adhering to the dietary restrictions of Greek Orthodox Lent. These restrictions emulate a typical Mediterranean diet, high in fruit, vegetables, cereals, olive oil and fish (Trichopoulou \& Vasilopoulou, 2000).

The second study by Linos et al. (1999) confirmed that higher intakes of olive oil reduced the risk of developing RA (OR 0.39 (95\% CI 0.19, 0.82), highest quartile of intake $v$. lowest quartile of intake), but greater fish consumption was no longer protective. It is unclear whether the study populations were mutually exclusive or whether some patients were studied twice.

Shapiro et al. (1996) measured past fish consumption in 324 incident RA cases and 1243 controls, all women. Participants were asked to report their 'usual' diet during the 5 years before the onset of symptoms using a selfadministered semi-quantitative food-frequency questionnaire. More than two servings of broiled or baked fish per week was associated with a reduced risk of RA compared with less than one serving, and the association was stronger in seropositive RA cases. The authors suggested that these findings confirmed their original hypothesis that higher intakes of $n$ - 3 PUFA from oily fish would reduce the risk of developing RA. However, there is some uncertainty as to whether the type of fish being eaten was in fact rich in oil. A further issue with this study was that the original food-frequency questionnaire was altered during the study, thereby introducing a potential systematic

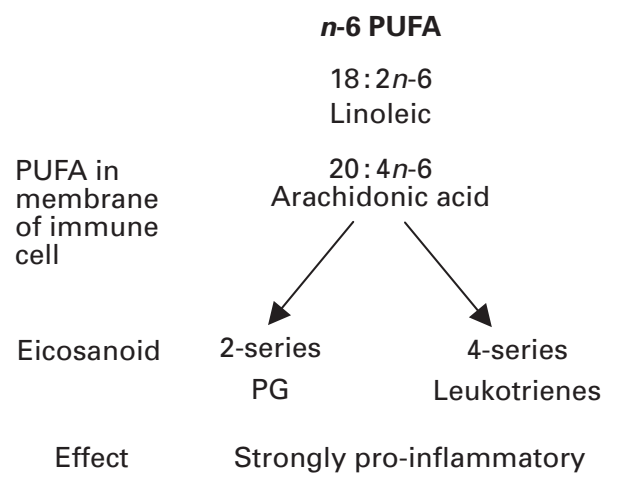

\section{n-3 PUFA}

$18: 3 n-3$
$\alpha$-Linolenic

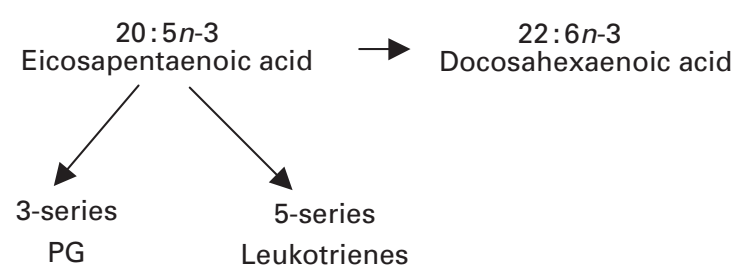

Weakly pro-inflammatory

Fig. 3. Formation of eicosanoids from $n-3$ and $n-6$ PUFA, and effects on inflammation. (Adapted from Buttriss, 1999.) 
bias in the data collection. Unlike the results from the Greek studies, no association was found with olive oil intake.

\section{Coffee and tea}

Despite there being no obvious biological reason for these beverages to have a role in the onset of arthritis, the association between coffee and tea consumption and RA has been explored in three population-based prospective studies that have found very different results (Heliövaara et al. 2000; Mikuls et al. 2002; Karlson et al. 2002). In the national health survey in Finland habitual coffee consumption was assessed at baseline by asking participants to estimate, on average, the number of cups of coffee they drank daily. Four or more cups of 'regular' coffee per d increased the risk of developing RA positive for RF, but not RF-negative RA (Heliövaara et al. 2000). In contrast, it was the decaffeinated coffee drinkers (four or more cups per d) participating in the Iowa Women's Health Study who had the greater risk of developing RA. Caffeinated coffee consumption had no association. However, in those women who drank most tea (more than three cups per d compared with none) there was a lower occurrence of RA. Again the associations were stronger in individuals who developed the RF-positive disease (Mikuls et al. 2002).

The Nurses' Health Study is an ongoing longitudinal cohort study of 121701 women, in which dietary data have been collected using multiple food-frequency questionnaires for $>15$ years. Karlson et al. (2002) found no significant association between drinking coffee or tea and the risk of RA.

\section{Alcohol}

Alcohol is thought to have effects on both hormonal and immunological systems, although the current knowledge is incomplete and often conflicting (Voigt et al. 1994; Cerhan et al. 2002). A biologically-plausible mechanism explaining the role that alcohol might have in the aetiology of RA has not been well established. In a hospital-based casecontrol study of 135 women with RA and 378 controls, those who consumed alcohol at least once per d halved their risk of RA onset (adjusted OR 0.54 (95\% CI 0.35, 0.82)) compared with non-drinkers (Hazes et al. 1990). However, current alcohol intake was assessed at their first visit to the clinic, after symptom onset. The mean time lag between patients' onset of symptoms and diet assessment was 1.5 years, so it is quite likely that RA cases could have modified their alcohol consumption before the assessment, which would not be representative of actual consumption before disease onset. A population-based case-control study (349 incident RA cases and 1457 controls; Voigt et al. (1994) also found a reduced, but not significant, risk of RA with higher alcohol intakes (more than fourteen drinks per week) in post-menopausal women (relative risk $0.5(95 \%$ CI $0.2,1.7))$. In contrast, no association was found between quantity or type of alcohol consumed and RA in the Iowa Women's Health Study (Cerhan et al. 2002).

\section{Meat}

Ecological studies are used to investigate associations between specific components of consumer food supply and disease prevalence at the national level, and are most useful for developing hypotheses for future investigation. In an ecological study of diet and RA (Grant, 2000) data from the national dietary supply for fifteen countries were derived from FAO food balance sheets and data on the prevalence of RA in these countries were taken from a compilation of prevalence-study results carried out between 1975 and 1988 . Meat and offal and meat fat, estimated 2 years before the dates of the prevalence studies, were found to have the highest association with the prevalence of RA $\left(r^{2} 0.649\right.$ and $r^{2} 0.679, P<0.001$ respectively). The correlations were stronger when the countries with the lowest meat consumption were removed from the analysis. Meat fat, nitrite and Fe content were considered as possible agents.

\section{Limitations of evidence}

The main limitation of the available evidence for a role of diet in the onset of RA is the variation in both outcome and exposure measures used across all studies. Both the original 1958 (Ropes et al. 1958) and the revised 1987 (Arnett et al. 1988) American College of Rheumatology criteria for the diagnosis of RA have been used, and both have well-recognised limitations in the diagnosis of RA in the clinical setting.

Food-frequency questionnaires were used in most studies (Linos et al. 1991, 1999; Shapiro et al. 1996; Cerhan et al. 2003), while other studies used dietary histories (Hall \& Myers, 1935; Bayles et al. 1943) or a single question built into an extensive lifestyle questionnaire (La Vecchia et al. 1998). Information relating to the validation process for most questionnaires was scarce. None of these studies used the food-diary method of assessment, the pros and cons of which have been debated for many years. However, recent support for its use in assessing diet more accurately has been provided (Bingham et al. 2003; Prentice, 2003). A number of studies were retrospective and, given that dietary recall is strongly influenced by current dietary intake, retrospective assessments may not be representative of diet before disease onset. Retrospective studies are also more likely to recruit longstanding prevalent cases, which make it difficult to distinguish whether diet is influencing the risk of developing RA or the progression of RA. It is important when investigating dietary risk factors for RA to examine the influence of diet unselected by disease severity, using an appropriate and validated method of dietary assessment. In order to achieve this objective cases should be recruited as soon as possible after symptom onset and, thus, cases of early undifferentiated IP can be compared with matched controls in the same population.

\section{Norfolk Arthritis Register-European Prospective Investigation of Cancer Diet Study}

This investigation has been made possible by the cooccurrence of two independent prospective studies of the 
same population, the Norfolk arm of the European Prospective Investigation of Cancer (EPIC)-Norfolk and the Norfolk Arthritis Register (NOAR), which have both been described in detail elsewhere (Symmons et al. 1994; Day et al. 1999). A prospective population-based nested case-control study was designed, in which the NOAR and EPIC-Norfolk databases were run against each other to identify incident cases of IP already participating in EPIC at the time of symptom onset ( $n$ 88). Cases were matched with two controls for gender, age (to within 3 years) and date of dietary assessment (to within 3 months). Diet was assessed using a $7 \mathrm{~d}$ 'estimated' food diary (Bingham et al. 1995) at baseline and the dietary intakes of the two groups were compared.

The results of this study suggest that participants with the lowest intakes of fruit and vegetables (lowest tertile compared with the highest tertile) have an increased risk of developing IP (adjusted OR 1.9 (95\% CI 1.0, 4.0)). However, participants in the lowest tertiles of vitamin $\mathrm{C}$ intake may be more than three times likely (adjusted OR $3.3(95 \%$ CI $1.4,7.9))$ to develop IP than those with greater intakes (Pattison et al. 2003). The lower intakes of $\beta$-carotene and vitamin $\mathrm{E}$ were associated with only a modest increase in risk of IP (OR $1.3(95 \%$ CI $0 \cdot 6,3 \cdot 2)$; OR $1.3(95 \%$ CI $0 \cdot 6,2 \cdot 7)$ respectively), whereas there was no relationship between retinol or Se intakes and risk of IP.

\section{Conclusions}

Fruit and vegetables appear to have an important role in the development of inflammatory arthritis (La Vecchia et al. 1998; Linos et al. 1999; Cerhan et al. 2003; Pattison et al. 2003). Few studies have specifically investigated the dietary intake of antioxidants and the risk of inflammatory arthritis, but lower intakes of vitamin $C$ and $\beta$-cryptoxanthin have been found in individuals who develop the condition (Shapiro et al. 1996; Cerhan et al. 2003; Pattison et al. 2003), and three studies of serum antioxidants have demonstrated low serum levels of antioxidants in samples taken before disease onset (Heliövaara et al. 1994; Comstock et al. 1997; Knekt et al. 2000). There are, of course, many other important antioxidants found in fruit and vegetables that have not been accounted for in the NOAR-EPIC collaboration so far. A future aim of this study is to address this particular aspect, given that it is possible that vitamin $\mathrm{C}$ is acting primarily only as a marker of fruit intake and that other micronutrients may be more relevant.

Other evidence of a role for diet in RA is limited to fish oil from dietary sources and olive oil. It is unlikely that coffee consumption itself has a direct effect on the initiation of RA, but greater coffee drinking is associated with cigarette smoking, which has been shown to be a risk factor for RA (Voigt et al. 1994; Symmons et al. 1997). Thus, coffee may influence risk indirectly. A low fruit and vegetable intake is also associated with smoking (Serdula et al. 1996). In the NOAR-EPIC collaboration smokers who developed IP also had lower intakes of fruit, vegetables and vitamin $C$ than their controls who smoked, despite total energy intake being similar across cases and controls.

It would seem reasonable to propose that poor diets may be responsible, in part, for increasing the risk of initiating inflammatory joint disease by removing powerful antioxidants that either protect against oxidative damage or prevent an efficient immune response, thus allowing the inflammatory process to progress unchecked. However, non-dietary factors are likely to be involved, and although diet may play an important role, this role would be as part of a complex pathway.

\section{Acknowledgements}

The NOAR is funded by the Arthritis Research Campaign. EPIC-Norfolk is supported by programme grants from the Cancer Research Campaign and the Medical Research Council with additional support from the British Heart Foundation, the Food Standards Agency, the Department of Health and the Europe Against Cancer programme of the Commission of the European Communities.

\section{References}

Abdel-Nasser AM, Rasker JJ \& Valkenburg HA (1997) Epidemiological and clinical aspects relating to the variability of rheumatoid arthritis. Seminars in Arthritis and Rheumatism 27, 123-140.

Arend WP (2001) The innate immune system in rheumatoid arthritis. Arthritis and Rheumatism 44, 2224-2234.

Arnett FC, Edworthy SM, Bloch DA, McShane DJ, Fries JF, Cooper NS et al. (1988) The American Rheumatism Association 1987 revised criteria for the classification of rheumatoid arthritis. Arthritis and Rheumatism 31, 315-324.

Bates CJ, Thurnham DI, Bingham SA, Margetts BM \& Nelson M (1997) Biochemical markers of nutrient intake. In Design Concepts in Nutritional Epidemiology, pp. 170-240 [BM Margetts and M Nelson, editors]. New York: Oxford University Press.

Bayles TB, Richardson H \& Hall FC (1943) The nutritional background of patients with rheumatoid arthritis. New England Journal of Medicine 229, 319-324.

Bingham SA, Cassidy A, Cole TJ, Welch A, Runswick SA, Black AE, Thurnham D, Bates C, Khaw K-T, Key TJA \& Day NE (1995) Validation of weighed records and other methods of dietary assessment using the $24 \mathrm{~h}$ urine nitrogen technique and other biological markers. British Journal of Nutrition 73, 531-550.

Bingham SA, Luben R, Welch A, Wareham N, Khaw K-T \& Day N (2003) Are imprecise methods obscuring a relation between fat and breast cancer? Lancet 362, 212-214.

Buttriss J (1999) n-3 Fatty acids and Health. BNF briefing paper, pp. 23-25. London: British Nutrition Foundation.

Cerhan JR, Saag KG, Criswell LA, Merlino LA \& Mikuls TR (2002) Blood transfusion, alcohol use, and anthropometric risk factors for rheumatoid arthritis in older women. Journal of Rheumatology 29, 246-254.

Cerhan JR, Saag KG, Merlino LA, Mikuls TR \& Criswell LA (2003) Antioxidant micronutrients and risk of rheumatoid arthritis in a cohort of older women. American Journal of Epidemiology 157, 345-354.

Comstock GW, Burke AE, Hoffman SC, Helzlsouer KJ, Bendich A, Masi AT, Norkus EP, Malamet RL \& 
Gershwin ME (1997) Serum concentrations of $\alpha$ tocopherol, $\beta$ carotene, and retinol preceding the diagnosis of rheumatoid arthritis and systemic lupus erythematosus. Annals of the Rheumatic Diseases 56, 323-325.

Darlington LG \& Stone TW (2001) Antioxidants and fatty acids in the amelioration of rheumatoid arthritis and related disorders. British Journal of Nutrition 85, 251-269.

Day N, Oakes S, Luben R, Khaw K-T, Bingham S, Welch A \& Wareham N (1999) EPIC-Norfolk: study design and characteristics of the cohort. British Journal of Cancer 80, Suppl. 1, 95-103.

Fortin PR, Lew RA, Liang MH, Wright EA, Beckett LA, Chalmers TC \& Soerling RI (1995) Validation of a metaanalysis: the effects of fish oil in rheumatoid arthritis. Journal of Clinical Epidemiology 48, 1379-1390.

Grant WB (2000) The role of meat in the expression of rheumatoid arthritis. British Journal of Nutrition 84, 589-595.

Hall FC \& Myers WK (1935) Diet in chronic arthritis. Archives of Internal Medicine 55, 403-410.

Halliwell B \& Gutteridge JMC (1999) Free radicals, other reactive species and disease. In Free Radicals in Biology and Medicine, 3rd ed., pp. 617-783. Oxford: Oxford University Press.

Halliwell B, Hoult JR \& Blake DR (1988) Oxidants, inflammation and anti-inflammatory drugs. FASEB Journal 2, 2867-2873.

Hazes JMW, Dijkmans BAC, Vandenbroucke JP, de Vries RRP \& Cats A (1990) Lifestyle and the risk of rheumatoid arthritis: cigarette smoking and alcohol consumption. Annals of the Rheumatic Diseases 49, 980-982.

Helgeland M, Svendsen E, Førre Ø \& Haughen M (2000) Dietary intake and serum concentrations of antioxidants in children with juvenile arthritis. Clinical and Experimental Rheumatology 18, 637-641.

Heliövaara M, Aho K, Knekt P, Impivaara O, Reunanen A \& Aromaa A (2000) Coffee consumption, rheumatoid factor, and the risk of rheumatoid arthritis. Annals of the Rheumatic Diseases 59, 631-635.

Heliövaara M, Knekt P, Aho K, Aaran R-K, Alfthan G \& Aromaa A (1994) Serum antioxidants and risk of rheumatoid arthritis. Annals of the Rheumatic Diseases 53, 51-53.

Karlson EW, Mandl LA, Aweh GN \& Grodstein F (2002) Coffee consumption and the risk of rheumatoid arthritis: Data from a large female cohort study. Arthritis and Rheumatism 46, Suppl., S456.

Kiziltunç A, Çogalgil S \& Cerrahoglu L (1998) Carnitine and antioxidants levels in patients with rheumatoid arthritis. Scandinavian Journal of Rheumatology 27, 441-445.

Knekt P, Heliövaara M, Aho K, Alfthan G, Marniemi J \& Aromaa A (2000) Serum selenium, serum alpha-tocopherol, and the risk of rheumatoid arthritis. Epidemiology 11, 402-405.

Kremer JM (1993) Nutrition and rheumatic diseases. In Textbook of Rheumatology, 4th ed., pp. 484-494 [WN Kelley, ED Harris, S Ruddy and CB Sledge, editors]. Philadelphia, PA: Saunders.

La Vecchia C, Decarli A \& Pagano R (1998) Vegetable consumption and risk of chronic disease. Epidemiology $\mathbf{9}$, 208-210.

Linos A, Kaklamani VG, Kaklamani E, Koumantaki Y, Giziaki E, Papazoglou S \& Mantzoros CS (1999) Dietary factors in relation to rheumatoid arthritis: a role for olive oil and cooked vegetables? American Journal of Clinical Nutrition 70, 1077-1082.

Linos A, Kaklamanis E, Kontomerkos A, Koumantaki Y, Gazi S, Vaiopoulos G, Tsokos GC \& Kaklamanis PH (1991) The effect of olive oil and fish consumption on rheumatoid arthritis - a case control study. Scandinavian Journal of Rheumatology 20, 419-426.

Lunec J, Halloran SP, White AC \& Dormandy TL (1981) Freeradical oxidation (peroxidation) products in serum and synovial fluid in rheumatoid arthritis. Journal of Rheumatology 8, 233-245.

Merry P, Winyard PG, Morris CJ, Grootveld M \& Blake DR (1989) Oxygen free radicals, inflammation, and synovitis: the current status. Annals of the Rheumatic Diseases 48, 864-870.

Mikuls TR, Cerhan JR, Criswell LA, Merlino L, Mudano AS, Burma M, Folsom AR \& Saag KG (2002) Coffee, tea, and caffeine consumption and risk of rheumatoid arthritis: Results from the Iowa Women's Health Study. Arthritis and Rheumatism 46, 83-91.

Pattison DJ, Silman AJ, Lunt M, Bunn D, Luben R, Welch A, Day NE \& Symmons DPM (2003) Low intake of vitamin C is associated with an increased risk of inflammatory polyarthritis. Rheumatology 42, Suppl. 1, 10.

Prentice R (2003) Dietary assessment and the reliability of nutritional epidemiology reports. Lancet 362, 182-183.

Rennie KL, Hughes J, Lang R \& Jebb SA (2003) Nutritional management of rheumatoid arthritis: a review of the evidence. Journal of Human Nutrition and Dietetics 16, 97-109.

Ropes MW, Bennett GA, Cobb S, Jacox R \& Jessar RA (1958) Revision of diagnostic criteria for rheumatoid arthritis. Bulletin of the Rheumatic Diseases 9, 175-176.

Serdula MK, Byers T, Mokdad AH, Simoes E, Mendlein JM \& Coates RJ (1996) The association between fruit and vegetable intake and chronic disease risk factors. Epidemiology 7, 161-165.

Shapiro JA, Koepsell TD, Voigt LF, Dugowson CE, Kestin M \& Nelson JL (1996) Diet and rheumatoid arthritis in women: a possible protective effect of fish consumption. Epidemiology 7, 256-263.

Silman AJ, MacGregor AJ, Thomson W, Holligan S, Carthy D, Farhan A \& Ollier WER (1993) Twin concordance rates for rheumatoid arthritis: results from a nationwide study. British Journal of Rheumatology 32, 903-907.

Symmons DPM, Bankhead CR, Harrison BJ, Brennan P, Barrett EM, Scott DGI \& Silman AJ (1997) Blood transfusion, smoking and obesity as risk factors for the development of rheumatoid arthritis. Results from a primary care-based incident case-control study in Norfolk, England. Arthritis and Rheumatism 40, 1955-1961.

Symmons DPM, Barrett EM, Bankhead CR, Scott DGI \& Silman AJ (1994) The incidence of rheumatoid arthritis in the United Kingdom: Results from the Norfolk Arthritis Register. British Journal of Rheumatology 33, 735-739.

Symmons D \& Harrison B (2000) Early inflammatory polyarthritis: results from the Norfolk Arthritis Register with a review of the literature. I. Risk factors for the development of inflammatory polyarthritis and rheumatoid arthritis. Rheumatology 39, 835-843.

Trichopolou A \& Vasilopoulou E (2000) Mediterranean diet and longevity. British Journal of Nutrition 84, S205-S209.

Voigt LF, Koepsell TD, Nelson JL, Dugowson CE \& Daling JR (1994) Smoking, obesity, alcohol consumption, and the risk of rheumatoid arthritis. Epidemiology 5, 525-532.

Young A, Cox N, Davies P, Devlin J, Emery P, Gallivan S, Gough A, James D, Prouse P, Williams P \& Winfield J (2000) How does functional disability in early RA affect patients and their lives? Results of 5 years of follow up in 732 patients from ERAS. Rheumatology 39, 603-611. 